\title{
Determination of the atmospheric point spread function by a parameter search
}

\author{
V. Krishnakumar ${ }^{\star}$ and P. Venkatakrishnan ${ }^{\star \star}$ \\ Indian Institute of Astrophysics, Bangalore 560034, India \\ Received April 28; accepted February 20, 1997
}

\begin{abstract}
The result of blind deconvolution is a reconstructed image that has non positive intensities. The number of these non positive pixels has been used as an estimator for the departure from a perfect reconstruction. Simulations of reconstruction of objects convolved with a kernel having one or two parameters are shown to demonstrate the efficiency of the estimator. We thus present a technique of determining the unknown parameters of the point spread function by searching for the point in parameter space with the lowest number of nonpositive pixels. It is also shown that the parameters of the convolving kernel can be obtained even in the presence of noise. This method was validated using a long exposure image of NGC 1409.
\end{abstract}

Key words: techniques: image processing - atmospheric effects - globular clusters: NGC 1409

\section{Introduction}

Turbulence in the earth's atmosphere degrades the true object intensity distribution of astronomical sources. The thermal gradients in the air produce random phase delays in the wavefront that cause blurring of images.

Usually all images which are exposed for several time scales of the atmospheric turbulence are classified as longexposure images. As a general rule of thumb, the exposure times in excess of a few hundredths of a second are considered as long exposure images. In long-exposure images the high spatial frequency information is attenuated because the recorded image is the source convolved with the time average of the point spread function (psf).

A straightforward method to measure the atmospheric psf is to measure the size of the intensity profile of an unresolved source close to the object under study. Here we assume that the medium through which the imaging is done behaves in the same way for both the object under study and the point source. If one has to get the true point spread function then the point source and the object under study should be within an isoplanatic patch.

\footnotetext{
* e-mail: krishna@iiap.ernet.in

$\star \star$ e-mail: pvk@iiap.ernet.in
}

For the sun, we do not have access to a point source for comparison. Furthermore, for extended sources like the sun, the atmospheric point spread function will not be the same on all parts of the image. We have a problem of an image for which each part of the object has been convolved with different point spread functions. Hence a single point spread function will not be a correct characterisation of the point spread function for an extended object.

Another technique (Collados 1987) of solar image reconstruction uses the limb of the moon in the photographs taken during partial solar eclipse. In the absence of earth's atmosphere the moon's limb would be seen as a sharp edge against the bright Sun's surface. When imaged using a ground based telescope, the moon's limb is blurred because of the atmospheric point spread function. The gradient of the blurred limb profile of the moon gives the point spread function of the telescope and atmosphere. The point spread function thus found is used for deconvolving the point spread function from the entire image. This point spread function can be used to remove blurring only near the limb of the moon and within the isoplanatic patch which encompasses the moon's limb. Use of this point spread function for deconvolution elsewhere in the image will not give true reconstruction.

Night time observers can have single stars for deconvolution. To get a reconstruction which is close to the true object intensity distribution, the star used for determining the point spread function of the atmosphere and the object under study have to be within the same isoplanatic patch. In the case of photometry of extended objects like clusters of stars, algorithms like Daophot are used (Stetson 1987) where nonisoplanaticity effects are not considered.

The conventional method is to make a gaussian fit to these observed profile and the full width at half maximum of the fitted gaussian is used to characterise the point spread function. This creates spurious features if the true point spread function is not a gaussian. In fact, there is theoretical and experimental evidence for the non gaussian nature of the atmospheric psf (Roddier 1980).

We propose a method of estimating the point spread function at any arbitrary part of an extended image based 
on a parameter search. We assume a class of convolving kernels involving one or two parameters and look for the number of zeros and negative pixel values in the reconstruction as a function of the parameters. We show that it is possible to retrieve the unknown parameters of the kernel. The technique proposed here has been rigourously tested on simulations and also on real images.

\section{Technique for recovering the point spread function}

The true object spectrum is convolved with the point spread function of the medium.

$<i(x, y)>=o(x, y) \times<h(x, y)>$

where $\langle i(x, y)\rangle$ is the time averaged intensity distribution, $o(x, y)$ is the true object intensity distribution, $<h(x, y)>$ is the time averaged intensity distribution and "*" denotes convolution. Performing Fourier transform on either side of Eq. (1), we get

$<I(u, v)>=O(u, v)<H(u, v)>$

where $\langle I(u, v)>, O(u, v),<H(u, v)>$ are the Fourier transforms of $\langle i(x, y)\rangle, o(x, y)<h(x, y)>$ respectively, and $u, v$ are the spatial frequency coordinates. To recover the true object spectrum $o(x, y)$, we perform inverse filtering on the degraded image. Therefore the true object intensity distribution will be

$O(u, v)=\frac{<I(u, v)>}{<H(u, v)>}$

Inverse transforming $O(u, v)$ we get $o(x, y)$. In our case $h(x, y)$ is not known. Guess psf is constructed and inverse filtering is done. Let $h_{\mathrm{g}}(x, y)$ be the guess psf. The Fourier transform of the guess psf is $H_{\mathrm{g}}(u, v)$. Using this psf we get,

$O_{\mathrm{g}}(u, v)=\frac{I(u, v)}{H_{\mathrm{g}}(u, v)}$.

The reconstructed image spectrum $o_{\mathrm{g}}(x, y)$ will be the inverse Fourier transform of $O_{\mathrm{g}}(u, v)$.

The point spread function of the atmosphere which blurs the object intensity distribution is (Tatarski 1961; Fried 1966)

$H(u, v)=\exp \left[-3.44\left(\frac{\lambda(u+v)}{r_{\mathrm{o}}}\right)^{\alpha}\right]$

where $u$ is the spatial frequency vector, $\lambda$ is the mean wavelength of observation, $r_{\mathrm{o}}$ is the Fried's parameter (seeing parameter) and $\alpha$ the power index which was derived to have a value of $5 / 3$ in the case of astronomical observations. In practice there could be deviations in the value of $\alpha$. The behaviour of the point spread function in the tail of the profile depends on $\alpha$ and $r_{\mathrm{o}}$ is a measure of the core of the point spread function profile. In our proposed technique we use the Fried's coherence function in its functional form but both $\alpha$ and $r_{\mathrm{o}}$ are left as free parameters.

The degraded image is deconvolved using a series of point spread functions with different $r_{\mathrm{o}}$ and $\alpha$. The number of elements $N$, equal to and less than zero is found in each reconstruction. In this two parameter space we search for the minimum of number of zeros and negative values. The corresponding $r_{\mathrm{o}}$ and $\alpha$ at which the minimum occurs are the true point spread function parameters.

In the presence of noise, Eq. (1) is written as

$<i(x, y)>=o(x, y) \times<h(x, y)>+n(x, y)$

where $n(x, y)$ is the noise in the image plane which gets added to the blurred object intensity distribution. Since noise is additive, it is not convolved with the atmospheric psf, but is effectively convolved with a delta function, which in turn, can be considered as a psf with very large Fried's parameter, say $r_{\mathrm{n}}$, where $r_{\mathrm{n}} \gg r_{\mathrm{o}}$

$<i(x, y)>=o(x, y) \times<h(x, y)>+n(x, y) \times h_{n}(x, y)(7)$

with $h_{\mathrm{n}}(x, y)$ approaching a delta function. For obtaining the parameters of the psf the above equation is Fourier transformed and inverse filtering is performed.

$<I(u, v)>=O(u, v)<H(u, v)>+N(u, v) H_{\mathrm{n}}(u, v) .(8)$

Inverse filtering,

$\frac{\langle I(u, v)>}{H_{\mathrm{g}}(u, v)}=\frac{O(u, v)<H(u, v)>}{H_{\mathrm{g}}(u, v)}+\frac{N(u, v) H_{\mathrm{n}}(u, v)}{H_{\mathrm{g}}(u, v)} .(9)$

This equation is inverse transformed and the number of non positive pixels are found. Similarly for other $r_{\mathrm{o}}$ values $H_{\mathrm{g}}(u, v)$ is constructed and the number of non positive pixels found. Since $r_{\mathrm{n}}$ is always greater than $r_{\mathrm{o}}$, the number of non-positive pixels $N$ contributed by the second term is not expected to go through a minimum. Therefore even in the presence of noise the minima in $N$ is expected to occur when the guess psf parameters matches with the true $r_{\mathrm{o}}$ and $\alpha$ values and hence $r_{\mathrm{o}}$ and $\alpha$ can be found by looking for the deepest minima in $N$ in the parameter space of $\alpha$ and $r_{\mathrm{o}}$.

This makes the proposed technique more general and could be used when the functional form of the point spread function of the intervening medium is of the Fried's coherence function type.

\section{Simulations}

\subsection{Object intensity distribution}

Simulations were carried out on single sources as well as on multiple sources. The technique has been tested on objects with intensity distribution with smooth edges as well as on objects with sharp edge. Here we discuss the case of an object with multiple sources and sharp edges. 


\subsection{Search for minima in $N$ in the absence of noise}

The true object consists of two sources with unequal intensities. The object plane has a plate scale of $\approx 0.06^{\prime \prime}$ per pixel. The angular size of source A and B is $0.4^{\prime \prime}$ and $0.5^{\prime \prime}$ respectively. The two sources are seperated by a angular distance of around $1^{\prime \prime}$. Figure 1 shows the true object intensity distribution and Fig. 2 is the atmospheric psf. The convolution of the object intensity distribution with the atmospheric psf yields the degraded image. Figure 3 is the convolved image. Fried derived the expression for the coherence function $H(u, v)$ and obtained a value of $5 / 3$ for $\alpha$. No departures are seen in the value of $\alpha=5 / 3$ under the conditions of astronomical observations. Assuming $\alpha$ is known, for each values of $r_{\mathrm{o}}$ the degraded image is deconvolved and $N$ is obtained. The plot of $N$ for various $r_{\mathrm{o}}$ is given in Fig. 4. We see that when the deconvolving psf's $r_{\mathrm{o}}$ is equal to the true $r_{\mathrm{o}}, N$ is a minimum.

However experimental evidence for departures from $5 / 3$ power law has been reported in the case of horizontal propogation near the ground (Bouricius \& Clifford 1970; Clifford et al. 1971; Buser 1971).

Therefore assuming that $\alpha$ is unknown, we do the following. For different values of $\alpha$, the reconstruction is done for a range of $r_{\mathrm{o}}$. Figure 5 is a plot of $N$ vs. $\alpha$ at a given $r_{\mathrm{o}}$. Here again we see that when the deconvolving psf's $\alpha$ becomes equal to the true psf's $\alpha, N$ goes to a minimum.

Now assuming both $\alpha$ and $r_{\mathrm{o}}$ are unknown, $N$ is found at each value of $\alpha$ and $r_{\mathrm{o}}$. Figure 6 is the surface plot of $N$ as a function of $r_{\mathrm{o}}$ and $\alpha$.

Wrong estimation of one of the parameters, $\alpha$ or $r_{\mathrm{o}}$ leads to inaccurate photometric values and also generates spurious features in the reconstructed image. Figure 7 is an example of an image degraded by a psf with $\alpha=1.67$, but reconstructed using $\alpha=1.7, r_{\mathrm{o}}=5.0 \mathrm{~cm}$ and Fig. 8 is reconstruction done with correct value of $\alpha$ but with a $r_{\mathrm{o}}=5.5 \mathrm{~cm}$ instead of the true $r_{\mathrm{o}}=5.0 \mathrm{~cm}$. We can clearly see spurious features in both the reconstructions.

In the presence of noise we need to reduce the grid size of the parameters in order to get the correct $r_{\mathrm{o}}$ or $\alpha$.

\subsection{Parametric search in the presence of noise}

The above simulations were repeated with different kinds of noise added to the blurred image.

Figure 9 is the image degraded by the atmospheric psf with $r_{\mathrm{o}}=5.0 \mathrm{~cm}$ and $\alpha=1.67$. A uniform distribution noise is added to the degraded image. The signal to noise ratio in all the noisy images is around 5.0.

Figure 10 is the plot of the number of non positive pixels $N$ for various values of Fried's parameter $r_{\mathrm{o}}$ in the presence of additive noise and Fig. 11 is the plot of the number of non positive pixels $N$ in each reconstructed image for different values of $\alpha$. In both the plots we see that at the minima in $N$ occurs at the true $\alpha$ and $r_{\mathrm{o}}$. Figure 12 is the surface plot of $\mathrm{N}$ as a function of $\alpha$ and $r_{\mathrm{o}}$. A search for the minima in $\mathrm{N}$ gives the exact values of $r_{\mathrm{o}}$ and $\alpha$ of the true psf.
Figure 13 is the convolved image to which zero mean Gaussian white noise has been added. Figure 14 is the plot of the number of non positive pixels $N$ in each reconstructed image for different values of Fried's parameter $r_{\mathrm{o}}$. Figure 15 and Fig. 16 are the plots of $N$ against $r_{\mathrm{o}}$ for reduced grid size of $r_{\mathrm{o}}, 0.5 \mathrm{~cm}$ and $0.1 \mathrm{~cm}$ respectively. It is seen that the minima in $N$ occurs at a $r_{\mathrm{o}}$ close to the true $r_{\mathrm{o}}$.

Figure 17 is the surface plot of the number of non positive pixels $N$ in the $r_{\mathrm{o}}, \alpha$ parameter space. The minima in $N$ gives the true $r_{\mathrm{o}}$ and $\alpha$.

Figure 18 is the image intensity distribution with additive Poisson noise. Figures 19, 20 and 21 are the plots of the number of non positive pixels $N$ against the Fried's parameter $r_{\mathrm{o}}$ with grid size in $r_{\mathrm{o}}$ equal to $1.0 \mathrm{~cm}, 0.5 \mathrm{~cm}$ and $0.1 \mathrm{~cm}$ respectively. We can see that for a grid size in $r_{\text {o }}$ equal to $1.0 \mathrm{~cm}$ the minima in $N$ occurs at $2.0 \mathrm{~cm}$. When the grid size is reduced to $0.5 \mathrm{~cm}$ the minima in $N$ shifts to $5.5 \mathrm{~cm}$ and the minima in $N$ stays at $5.5 \mathrm{~cm}$ when the grid size is reduced to $0.1 \mathrm{~cm}$. Hence in the presence of noise reduction of grid size helps to identify the parameters $r_{\mathrm{o}}$ and $\alpha$ more accurately.

Figure 22 is the surface plot of $N$ in the parameter space of $r_{\mathrm{o}}$ and $\alpha$. The minima in $N$ occurs at the true $r_{\mathrm{o}}$ and $\alpha$.

However, any deconvolution performed on these images will not give the correct reconstruction unless the noise is filtered.

\section{Conclusions}

The technique we have proposed in this paper is very general and is applicable for images of arbitrary shape. It can also provide a decent reconstruction for features that are several hundred times more intense than noise. However, the problem of image reconstruction still remains for low contrast features with noise. We believe that the knowledge of the actual point spread function, as gained by the parametric search described in this paper, will provide tight constraints on iterative deconvolution schemes for very noisy images which can start with a guess for the noise distribution and converge to the true distribution of noise. This belief is yet to be verified. On the other hand, there are a number of applications where the determination of the psf itself would be of vital importance. One example is the determination of daytime "seeing" at a new astronomical site. All that would be required is a sequence of long exposure pictures of the sun taken through a filter of reasonable bandwidth like $5 \mathrm{~nm}$ or $10 \mathrm{~nm}$. Another example is the need to determine the broadening mechanisms of spectral lines. We hope that this technique would be able to detect small departures from a gaussian profile, which has great implications for the detection of non-thermal or supra-thermal distributions. The quality of spectra that are expected from SOHO, for example, would perhaps be good enough for such investigations. 
This technique was used to estimate the Fried's parameter in the image of the globular cluster. The results are discussed in Appendix 1. The main result in appendix is the practical demonstration of the successful working of the parameter search method. The choice of a star cluster to test the method is very appropriate because the entire object occupies several isoplanatic patches while each stellar member of the cluster provides a point source to provide an unambiguous psf. We see here that the conventional method of fitting a Gaussian profile and FWHM of the Gaussian fit used as a measure of the Fried's parameter matches well with the Fried's parameter obtained using the parameter search method.

Acknowledgements. We would like to thank Prof. Ram Sagar and Mr. Alok Gupta for providing with the image of the globular cluster NGC 1409. We would like to thank the anonymous referee for useful suggestions and comments.

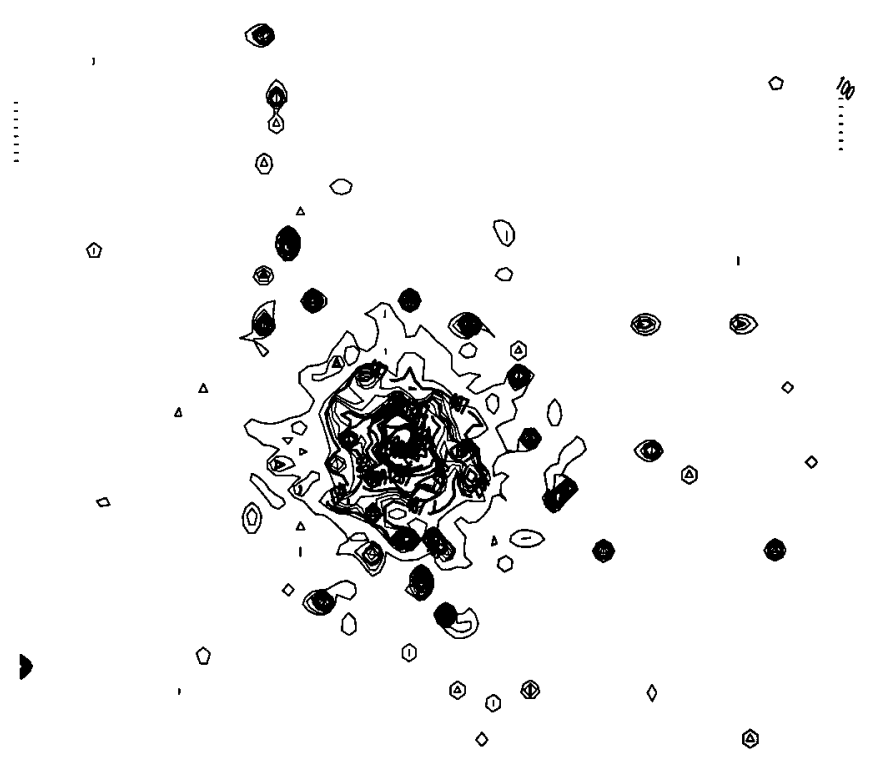

(1)

Fig. 1. A) Contour map of globular cluster NGC 1409 observed using the $2.34 \mathrm{~m}$ optical telescope at Vainu Bappu Observatory, Kavalur. The mean wavelength of observation is $5656 \AA$

\section{Appendix 1}

\section{Parametric search for Fried's parameter on globular cluster}

We compare the results obtained using parameter search method with the Fried's parameter obtained using a conventional method and show that the results obtained using two different methods are in good agreement.

\section{Parametric search on a globular cluster (NGC 1409)}

We were looking for an image with many point sources in order to estimate the Fried's parameter using conventional methods like fitting a Gaussian and using its FWHM as a measure of the Fried's parameter $r_{\mathrm{o}}$. We chose an image of the globular cluster NGC 1409 (observed by Prof. Ram Sagar and Mr. Alok Gupta at the Vainu Bappu Observatory using the $2.34 \mathrm{~m}$ Optical telescope) for this purpose.

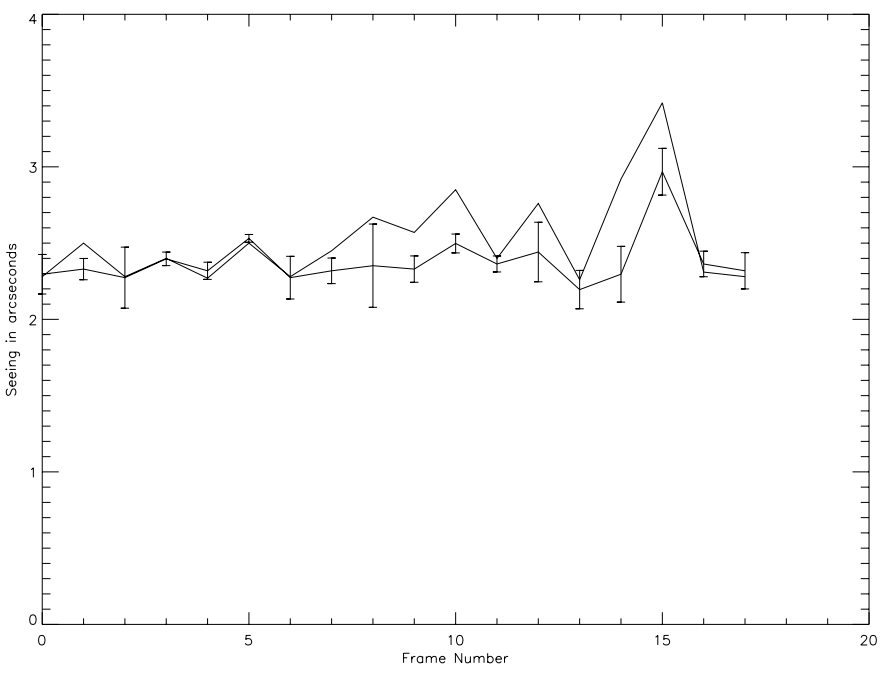

Fig. 2. A) Plot of seeing estimate obtained using parametric search and seeing estimated using average of FWHM $\times 1.12$ (the factor of 1.12 is multiplied with the FWHM to take care of the non Gaussian nature of the Fried's coherence function)

The image is a $1024 \times 1024$ pixel CCD image with a plate scale of $0.6 \%$. The mean wavelength of observation is $5656 \AA$. The contour of the globular cluster NGC 1409 is shown in Fig. 1A. The field is approximately $10 \operatorname{arcmin} \times 10 \operatorname{arcmin}$. The isoplanaticity of the sky is not expected to be of that size. We divide the observed image into smaller sections, each of $50 \operatorname{arcsec} \times 50$ arcsec. The parametric search algorithm is run on each such small sub images and the Fried's parameter is estimated. Since the plate scale and the mean wavelength of observation is known, the Fried's parameter can be now converted to "seeing" in arcseconds in the real domain.

On each subimage there are several point sources. A best fit Gaussian is made for each point source in the sub image and the average FWHM is estimated. This directly gives the seeing in pixel units. The plate scale is known and hence seeing is calculated in terms of arcseconds. A factor of 1.12 needs to be multiplied to these values to take care of the non-gaussian nature of the Fried's coherence function.

Figure 2A gives the plot of the seeing estimated both using the parameter search method and also using the 
average FWHM of the Gaussians (corrected for the nongaussian nature of the atmospheric psf) in each sub image. We see a similar trend in the seeing estimated using the two different methods. The values obtained using the two different methods agree within the error limits.

\begin{tabular}{|c|c|c|}
\hline Frame & $\begin{array}{c}\text { Seeing estimated using } \\
\text { Average FWHM } \\
\text { of Gaussian fit } \\
\times 1.12 \\
\text { (in arcsec) }\end{array}$ & $\begin{array}{c}\text { Seeing estimated using } \\
\text { Parametric Search }\end{array}$ \\
\hline 1 & $2.29 \pm 0.22$ & \\
2 & $2.32 \pm 0.12$ & 2.28 \\
3 & $2.27 \pm 0.33$ & 2.50 \\
4 & $2.40 \pm 0.07$ & 2.28 \\
5 & $2.32 \pm 0.09$ & 2.40 \\
6 & $2.53 \pm 0.04$ & 2.27 \\
7 & $2.27 \pm 0.23$ & 2.50 \\
8 & $2.32 \pm 0.14$ & 2.28 \\
9 & $2.35 \pm 0.46$ & 2.45 \\
10 & $2.33 \pm 0.14$ & 2.67 \\
11 & $2.50 \pm 0.10$ & 2.57 \\
12 & $2.36 \pm 0.09$ & 2.85 \\
13 & $2.44 \pm 0.33$ & 2.40 \\
14 & $1.96 \pm 0.21$ & 2.76 \\
15 & $2.05 \pm 0.30$ & 2.26 \\
16 & $2.65 \pm 0.26$ & 2.92 \\
17 & $2.11 \pm 0.14$ & 3.42 \\
18 & $2.50 \pm 0.20$ & 2.31 \\
& & 2.85 \\
\hline
\end{tabular}

\section{References}

Bouricius G.M.B., Clifford.S.F., 1970, J. Opt. Soc. Am. 60, 1484

Buser R.G., 1971, J. Opt. Soc. Am. 61, 488

Clifford S.F., Bouricius G.M.B., Ochs G.R., Ackley M.H., 1971, J. Opt. Soc. Am. 61, 1279

Clifford S.F., Ochs G.R., Lawerence R.S., 1974, J. Opt. Soc. Am. 64, 148

Fried D.L., 1966, J. Opt. Soc. Am. 56, 1372

Goodman J.W., 1985, Statistical Opt. McGraw Hill, New York

Peter B.Stetson., 1987, PASP 99, 191

Roddier., The Effects of Atmospheric Turbulence in Optical Astronomy. In: Wolf E. (ed.) Prog. Opt., p. 281

Tatarski V.I., 1961, Wave Propogation in Turbulent Medium. McGraw Hill Book Co., New York

Collados M., Vasquez M., 1987, A\&A 180, 223-228 